\title{
Radical Phosphate Transfer Mechanism for the Thiamin Diphosphate- and FAD-Dependent Pyruvate Oxidase from Lactobacillus plantarum. Kinetic Coupling of Intercofactor Electron Transfer with Phosphate Transfer to Acetyl-thiamin Diphosphate via a Transient FAD Semiquinone/Hydroxyethyl-ThDP Radical Pair
}

\author{
Kai Tittmann,*,‡ Georg Wille, ${ }^{\ddagger}$ Ralph Golbik, ${ }^{\ddagger}$ Annett Weidner, ${ }^{\ddagger}$ Sandro Ghisla, ${ }^{\S}$ and Gerhard Hübner ${ }^{\ddagger}$ \\ Institut für Biochemie, Martin-Luther-Universität Halle-Wittenberg, Kurt-Mothes-Strasse 3, D-06120 Halle/Saale, Germany, and \\ Biologische Fakultät, Universität Konstanz, Germany
}

\begin{abstract}
The thiamin diphosphate (ThDP)- and flavin adenine dinucleotide (FAD)-dependent pyruvate oxidase from Lactobacillus plantarum catalyses the conversion of pyruvate, inorganic phosphate, and oxygen to acetyl-phosphate, carbon dioxide, and hydrogen peroxide. Central to the catalytic sequence, two reducing equivalents are transferred from the resonant carbanion/enamine forms of $\alpha$-hydroxyethylThDP to the adjacent flavin cofactor over a distance of approximately $7 \AA$, followed by the phosphorolysis of the thereby formed acetyl-ThDP. Pre-steady-state and steady-state kinetics using time-resolved spectroscopy and a ${ }^{1} \mathrm{H}$ NMR-based intermediate analysis indicate that both processes are kinetically coupled. In the presence of phosphate, intercofactor electron-transfer (ET) proceeds with an apparent first-order rate constant of $78 \mathrm{~s}^{-1}$ and is kinetically gated by the preceding formation of the tetrahedral substrateThDP adduct 2-lactyl-ThDP and its decarboxylation. No transient flavin radicals are detectable in the reductive half-reaction. In contrast, when phosphate is absent, ET occurs in two discrete steps with apparent rate constants of 81 and $3 \mathrm{~s}^{-1}$ and transient formation of a flavin semiquinone/hydroxyethyl-ThDP radical pair. Temperature dependence analysis according to the Marcus theory identifies the second step, the slow radical decay to be a true ET reaction. The redox potentials of the $\mathrm{FAD}_{\mathrm{ox}} / \mathrm{FAD}_{\mathrm{sq}}\left(E_{1}=-37 \mathrm{mV}\right)$ and $\mathrm{FAD}_{\mathrm{sq}} / \mathrm{FAD}_{\text {red }}\left(E_{2}=-87 \mathrm{mV}\right)$ redox couples in the absence and presence of phosphate are identical. Both the Marcus analysis and fluorescence resonance energy-transfer studies using the fluorescent N3'pyridyl-ThDP indicate the same cofactor distance in the presence or absence of phosphate. We deduce that the exclusive $10^{2}-10^{3}$-fold rate enhancement of the second ET step is rather due to the nucleophilic attack of phosphate on the kinetically stabilized hydroxyethyl-ThDP radical resulting in a low-potential anion radical adduct than phosphate in a docking site being part of a through-bonded ET pathway in a stepwise mechanism of ET and phosporolysis. Thus, $L p$ POX would constitute the first example of a radical-based phosphorolysis mechanism in biochemistry.
\end{abstract}

Pyruvate oxidase from Lactobacillus plantarum ( $\mathrm{LPOX},{ }^{1}$ EC 1.2.3.3) belongs to a superfamily of enzymes that utilize the cofactor thiamin diphosphate (ThDP), the biologically active derivative of vitamin $\mathrm{B}_{1}$. In addition to ThDP, $L p \mathrm{POX}$ contains a flavin adenine dinucleotide (FAD) that is positioned at a distance of approximately $7 \AA$ from the thiamin cofactor (Figure 1) (1).

Pyruvate oxidase (POX) catalyses the conversion of pyruvate, inorganic phosphate, and oxygen to the high-energy

* To whom correspondence should be addressed: E-mail: kai@ bc.biochemtech.uni-halle.de. Telephone: ++49-345-5524887. Fax: $++49-345-5527011$.

Martin-Luther-Universität Halle-Wittenberg.

$\S$ Universität Konstanz.

${ }^{1}$ Abbreviations: ET, electron transfer; POX, pyruvate oxidase; $L p$ POX, pyruvate oxidase from L. plantarum; EcPOX, pyruvate oxidase from E. coli; EcAHAS II, acetohydroxyacid synthase isozym II from E. coli; $\mathrm{ThDP}$, thiamin diphosphate; $\mathrm{ThDP}^{-}$, carbanion form of $\mathrm{ThDP}$; LThDP, 2-lactyl-ThDP; HEThDP ${ }^{-}$, carbanion/enamine form of 2-hydroxyethyl-ThDP; AcThDP, 2-acetyl-ThDP; FAD, flavin adenine dinucleotide; $\mathrm{FAD}_{\mathrm{ox}}, \mathrm{FAD}$ in the oxidized state; $\mathrm{FAD}_{\mathrm{sq}}$, one-electronreduced semiquinone form of $\mathrm{FAD} ; \mathrm{FAD}_{\text {red }}$, two-electron-reduced form of FAD.

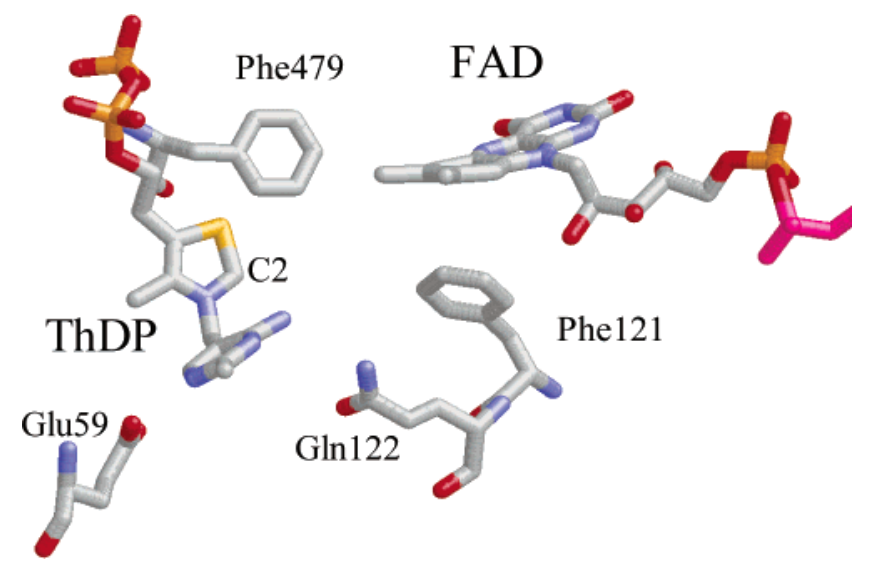

FIGURE 1: Active-site structure of $L p P O X$ showing the cofactors ThDP and FAD and selected amino acid residues (1).

metabolite acetyl phosphate, carbon dioxide, and hydrogen peroxide (2). Catalysis can be assumed to follow the typical Breslow mechanism of ThDP enzymes (Scheme 1). After ionization of the acidic $\mathrm{C} 2-\mathrm{H}$ of the thiazolium ring, pyruvate enters the active site where it reacts with the C2 
Scheme 1: Suggested Catalytic Cycle of $L p P O X$ with Key Intermediates Invoked in Oxidative Decarboxylation of Pyruvate ${ }^{a}$

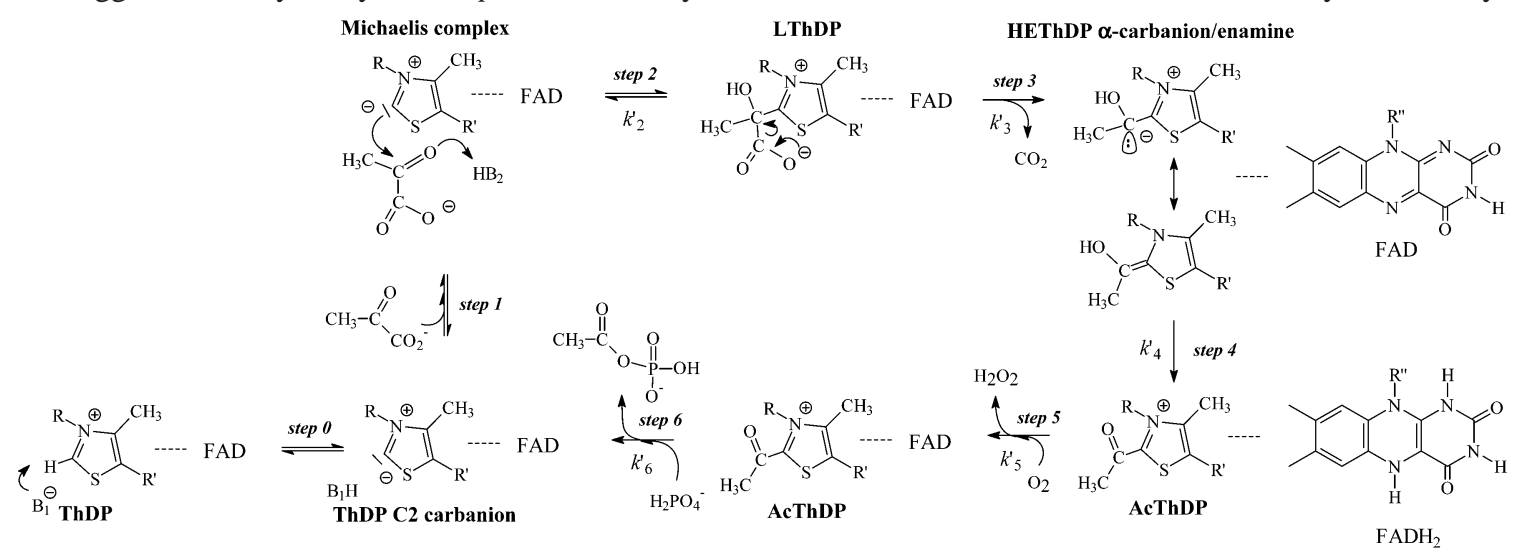

${ }^{a}$ Note that the fully reduced FAD shown here as $\mathrm{FADH}_{2}$ may occur in the anionic $\mathrm{FADH}^{-}$form.

Scheme 2: Reactivity of 2-Acetyl-thiazolium Salts and 2-Acetyl-ThDP with Phosphate Anions and Hydroxide Ions According to Refs 3 and 4
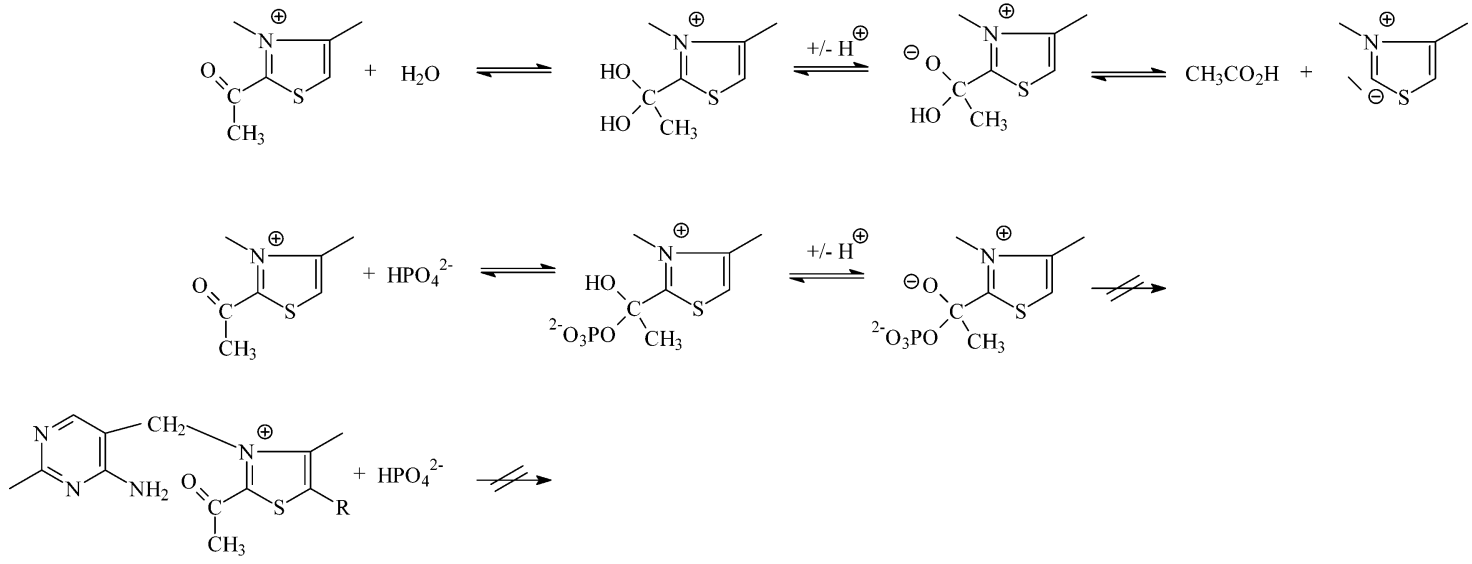

carbanion, leading to the formation of the tetrahedral substrate-ThDP adduct 2-lactyl-ThDP (LThDP). After decarboxylation of LThDP, two reducing equivalents are transferred from the resonant carbanion/enamine forms of 2-hydroxyethyl-ThDP (HEThDP) to the flavin cofactor, yielding 2-acetyl-ThDP (AcThDP) and the two-electron reduced flavin $\left(F A D_{\text {red }}\right)$. Finally, $F A D_{\text {red }}$ is reoxidized by molecular oxygen to give hydrogen peroxide and FAD, whereby AcThDP is cleaved phosphorolytically to acetyl phosphate and ThDP.

Model studies on the chemistry of the elusive AcThDP intermediate by Frey and co-workers revealed that AcThDP is subject to hydrolysis; however, phosphate anions do not even form covalent adducts with AcThDP (Scheme 2) (3). To the contrary, phosphate could add to 2-acetyl-thiazolium salts, but the resultant adduct rather reverts to the reactants as opposed to decay to the products in aqueous solution. This is because phosphate is the better leaving group compared to the thiazolium $\mathrm{C} 2$ ylide (4). A sufficient driving force for expulsion of the ylide is only provided after addition of an hydroxide ion.

From a chemical point of view, it is thus exciting to learn how $L p$ POX catalyses an efficient phosphate transfer from the solvent to AcThDP and circumvents the competing hydrolysis of this key intermediate. Our present studies provide evidence that $L p P O X$ makes use of flavin-mediated radical chemistry. Our results are consistent with a mechanism that involves a stepwise transfer of the two electrons from the HEThDP carbanion/enamine to FAD and nucleophilic attack of phosphate on the transiently formed and kinetically stabilized hydroxyethyl-ThDP radical. The presumably low potential of the anion radical adduct formed in the process could then provide a sufficient driving force to account for the observed forward commitment in $L p P O X$ catalysis. Aside from pyruvate ferredoxin oxidoreductase (PFOR) $(5,6)$, this would constitute the second case of a ThDP enzyme that relies on radical chemistry for catalysis, mirroring the chemical versatility and catalytic potential of the thiamin cofactor.

\section{MATERIALS AND METHODS}

Chemicals and Reagents. Sodium pyruvate, $\beta$-D glucose, glucose oxidase, benzyl viologen, ThDP, and FAD were purchased from Sigma. Sodium dithionite was supplied from Merck. All redox dyes (indigo disulfonate, cresyl violet, and phenosafranine) used in this study were a generous gift from Dr. Loredano Pollegioni (University of Insumbria, Italy). The coenzymatically inactive ThDP analogue N3'-pyridyl-ThDP was synthesized according to ref 7 . All other chemicals were of analytical grade and purchased from commercial sources. Quartz double-distilled water was used throughout the experiments.

Expression and Protein Purification. Expression and purification of $L p \mathrm{POX}$ were performed as described in ref 8. Apo-enzyme preparation was carried out according to the protocol given in ref 9 . 
Acetohydroxyacid synthase isozyme II from Escherichia coli (EcAHAS II, EC 4.1.3.18) was expressed and purified as described in ref 10 . The purification of pyruvate oxidase from $E$. coli (EcPOX, EC 1.2.3.3) will be described in full detail elsewhere (Weidner et al., manuscript in preparation).

Determination of Protein Concentration. The purified proteins were quantified by absorbance using an extinction of $1.65 A_{278} \mathrm{~nm} \mathrm{mg} \mathrm{mL} \mathrm{cm}^{-1}$ (LpPOX), $0.9 A_{280 \mathrm{~nm} \mathrm{mg}} \mathrm{mL}^{-1}$ $\mathrm{mL} \mathrm{cm}{ }^{-1}$ (EcAHAS II), or by the method of Bradford $(E c \mathrm{POX})(11)$.

Steady-State Kinetics. The enzymatic activity of LpPOX was determined in the presence of $50 \mathrm{mM}$ pyruvate in 0.2 $\mathrm{M}$ air-saturated potassium phosphate buffer at $\mathrm{pH} 6.0$ at 25 ${ }^{\circ} \mathrm{C}$. Two different assays were used to determine the specific activity. Oxygen consumption during turnover was directly measured using a temperature-controlled oxygen electrode (Rank Brothers, Cambridge, U.K.). The reduction of the artificial electron acceptor 2,6-dichlorophenolindophenol (DCPIP) in the course of substrate turnover was followed by the decrease of absorbance at $600 \mathrm{~nm}\left(\epsilon_{\text {DCPIP }}=17.7 \mathrm{~cm}^{-1}\right.$ $\left.\mathrm{mM}^{-1}\right)$.

Rapid Reaction Kinetics. The reduction of the enzymebound flavin in $L p \mathrm{POX}$ during turnover was kinetically analyzed with a stopped-flow spectrophotometer (Applied Photophysics SX18 MV, U.K.) using the intrinsic FAD absorbance at $457 \mathrm{~nm}$. Reactions were carried out under aerobic and anaerobic conditions (12), either in $0.2 \mathrm{M}$ potassium phosphate buffer at $\mathrm{pH} 6.0$ or in $0.1 \mathrm{M}$ Mes $/ \mathrm{NaOH}$ at pH 6.0 and $25^{\circ} \mathrm{C}$. Typically, $15-20 \mu \mathrm{M} L p \mathrm{POX}$ (activesite concentration) were reacted with varied concentrations of pyruvate $(0.5-50 \mathrm{mM})$.

Kinetic traces obtained under aerobic conditions were analyzed according to the method of Gibson et al. (13). In brief, the area under the progress curve under steady-state conditions can be assumed to be proportional to the concentration of oxygen in the solution as follows:

$$
\left[\mathrm{O}_{2}\right]_{\mathrm{total}}=\alpha\left[\int_{t_{0} \mathrm{SS}}^{t_{x}} A_{\mathrm{FAD}}(t) \mathrm{d} t-A_{\mathrm{FAD}}\left(t_{x}\right)\left(t_{x}-t_{0}^{\mathrm{SS}}\right)\right]
$$

where $t_{0}^{\mathrm{SS}}$ is the starting point of the steady state, $t_{x}$ defines the end of the reaction, $A_{\mathrm{FAD}}$ is the absorbance of the enzymebound flavin at the chosen analytical wavelength $(457 \mathrm{~nm}$ for $L p \mathrm{POX}$ ), and $\alpha$ is a proportionality factor.

This correlation allows one to calculate $\mathrm{d}\left[\mathrm{O}_{2}\right] / \mathrm{d} t$ and the remaining oxygen concentration in the solution at any time point of the reaction according to eq 2

$$
\begin{aligned}
& \left.v(\text { [pyruvate],[phosphate }],\left[\mathrm{O}_{2}\right]\right)= \\
& \frac{\int_{t_{1}}^{t_{2}} A_{\mathrm{FAD}}(t) \mathrm{d} t-A_{\mathrm{FAD}}\left(t_{x}\right)\left(t_{2}-t_{1}\right)}{\int_{t_{0} \mathrm{SS}}^{t_{x}} A_{\mathrm{FAD}}(t) \mathrm{d} t-A_{\mathrm{FAD}}\left(t_{x}\right)\left(t_{x}-t_{0} \mathrm{SS}\right)} \cdot \frac{\left[\mathrm{O}_{2}\right]_{\text {total }}}{\left(t_{2}-t_{1}\right)}
\end{aligned}
$$

Kinetic data were analyzed using Kaleidagraph.

The reductive half-reaction of $L p \mathrm{POX}$, which comprises the formation of the Michaelis complex with the substrate, carbonyl addition of pyruvate to the ThDP C2 ylide, decarboxylation of the resultant LThDP intermediate, and transfer of two reducing equivalents from the HEThDP carbanion/enamine to FAD (steps 1-4 in Scheme 1), was analyzed under anaerobic conditions as described before (12). At pyruvate saturation, the apparent rate constant of FAD reduction $\left(k_{\mathrm{obs}}\right)$ is kinetically related to the unimolecular net rate constants of elementary steps (see Scheme 1) as follows:

$$
\frac{1}{k_{\mathrm{obs}}}=\frac{1}{k_{2}^{\prime}}+\frac{1}{k_{3}^{\prime}}+\frac{1}{k_{4}^{\prime}}
$$

As we have shown recently (12), the reversible reaction of pyruvate with the enzyme (step 1 in Scheme 1) to form the Michaelis complex is not rate-limiting for the reduction when pyruvate is saturating. Under these conditions, only steps $2-4$ are kinetically significant for the reduction reaction.

In addition to the single-wavelength measurements, timeresolved absorbance spectra were recorded with a similar stopped-flow spectrometer equipped with a diode array detector (J\&M TIDAS). The wavelength range was from 300 to $700 \mathrm{~nm}$, and the aquisition time of the detector was up to $0.7 \mathrm{~ms} / \mathrm{spectrum}$. All spectra are corrected for buffer and substrate absorbance.

The reduction of enzyme-bound FAD in EcPOX was analyzed in the same way and under the same conditions as described for $L p P O X$. The kinetic study of electron transfer (ET) in EcAHAS II was carried out in $0.1 \mathrm{M}$ potassium phosphate buffer at $\mathrm{pH} 7.6$ and in $0.1 \mathrm{M}$ Tris/ $\mathrm{HCl}$ at $\mathrm{pH}$ 7.6.

Temperature Analysis According to the Marcus Theory and the Transition-State Theory. The apparent rate constants $\left(k_{\mathrm{obs}}\right)$ of FAD reduction in the presence and absence of phosphate were analyzed according to the ET theory established by Marcus and Sutin (14). In this theory, the rate constant of a nonadiabatic ET $\left(k_{\mathrm{ET}}\right)$ is related to the temperature of the reaction and a series of parameters as follows (eqs 4 and 5):

$$
\begin{gathered}
k_{\mathrm{ET}}=\frac{4 \pi^{2}\left(H_{\mathrm{AB}}\right)^{2}}{h \sqrt{4 \pi \lambda R T}} \exp \left[-\left(\Delta G^{\circ}+\lambda\right)^{2}\right] / 4 \lambda R T \\
k_{\mathrm{ET}}=k_{0} \exp \left(-\beta\left(r-r_{0}\right) \exp \left[-\Delta G^{\circ}+\lambda\right)^{2}\right] / 4 \lambda R T
\end{gathered}
$$

where $h$ is Planck's constant $\left(6.62 \times 10^{-34} \mathrm{~J} \mathrm{~s}\right), R$ is the gas constant $\left(8.314 \mathrm{~J} \mathrm{~K}^{-1} \mathrm{~mol}^{-1}\right), k_{0}$ is the characteristic frequency of the nuclei $\left(10^{13} \mathrm{~s}^{-1}\right)$, and $r_{0}$ is the close contact distance (assigned as $3 \AA$ ). The Marcus parameters describing ET are the coupling constant $H_{\mathrm{AB}}$ as a measure of the degree of wave function overlap between acceptor and donor, the reorganizational energy $\lambda$, the standard free energy of the ET reaction $\Delta G^{\circ}$, the electronic decay factor $\beta$, which is related to the nature of the intervening medium, and the interatomic edge-edge distance $r$ between the donor and acceptor. The apparent rate constants of FAD reduction at different temperatures were first fitted to eq 4, thereby obtaining $H_{\mathrm{AB}}, \Delta G^{\circ}$, and $\lambda$. The calculated reorganizational energy and standard free-energy change were then used to fit the same data to eq 5 to obtain estimates of the electronic decay factor $\beta$ and the interatomic distance between the donor and acceptor. Fitting of the apparent rate constants is helpful to gather information whether the rate-determining process is a true or gated ET reaction. In contrast to nonadiabatic (true) ET reactions, the fitted value of $H_{\mathrm{AB}}$ of a gated ET will exceed the nonadiabatic limit of approximately $80 \mathrm{~cm}^{-1}$ (15). Moreover, fitting the data of a coupled ET to eq 5 will likely give unreasonable values of the edge-edge distances of the donor and acceptor. A detailed description of the 
theory and its application to biological systems is given in refs $14-18$ and references therein.

In case the Marcus theory could not adequately describe the observed process, the transition-state theory was applied to obtain the activation enthalpy $\Delta H^{\#}$ and the activation entropy $\Delta S^{\#}$ for the reaction. Plots of the ln of the observed rate constants $k_{\text {obs }}$ were fitted versus $1 / T$ to eq 6

$$
\ln \left(\frac{k_{\mathrm{obs}} h}{k_{\mathrm{B}} T}\right)=\frac{-\Delta H^{\#}}{R T}+\frac{\Delta S^{\#}}{R}
$$

where $h$ is Planck's constant, $k_{\mathrm{B}}$ is the Boltzmann constant $\left(1.38 \times 10^{-23} \mathrm{~J} \mathrm{~K}^{-1}\right), R$ is the gas constant, and $T$ is the absolute temperature.

Intermediate Analysis by Acid-Quench ${ }^{1} H$ NMR Spectroscopy and Calculation of the Unimolecular Net Rate Constants of All Elementary Steps. For the quantitative analysis of the distribution of covalent ThDP intermediates, we employed a combined acid-quench/ ${ }^{1} \mathrm{H}$ NMR method (19) that makes use of the slightly different chemical shifts of the $\mathrm{C}^{\prime}-\mathrm{H}$ proton resonances of the isolated cofactor intermediates LThDP (7.27 ppm), HEThDP (7.33 ppm), and AcThDP (7.35 ppm) at low $\mathrm{pH}$. Because some proton signals of FAD interfere with the $\mathrm{C6}^{\prime}-\mathrm{H}$ resonance of unsubstituted ThDP (8.01 ppm), we used the $\mathrm{C} 2-\mathrm{H}$ signal of ThDP for analysis in that case. A solution of $15 \mathrm{mg} / \mathrm{mL} L p P O X(230 \mu \mathrm{M}$ active sites) was mixed with $100 \mathrm{mM}$ pyruvate in a $1+1$ mixing ratio in a chemical quench flow apparatus (RQF-3, Kintek Corp., TX). The reaction was allowed to age for defined reaction times ranging from $2 \mathrm{~ms}$ to $10 \mathrm{~s}$. The reaction was then stopped by addition of an acidic quench solution containing $12.5 \%(\mathrm{w} / \mathrm{v})$ trichloroacetic acid (TCA) and $1 \mathrm{M}$ $\mathrm{DCl}$ in $\mathrm{D}_{2} \mathrm{O}$. After the denatured protein was removed by centrifugation, the isolated intermediates were analyzed by ${ }^{1} \mathrm{H}$ NMR spectroscopy (19).

The concentration ratio of the different ThDP intermediates under true steady-state conditions may then be utilized to calculate the unimolecular forward (net) rate constants. As given in eq $3, k_{2}^{\prime}, k_{3}^{\prime}$, and $k_{4}^{\prime}$ can be related to the apparent rate constant $k_{\mathrm{obs}}$ of FAD reduction obtained by the stoppedflow experiments under anaerobic conditions. When the ThDP intermediates are co-isolated in the steady-state, the following correlations hold true at pyruvate saturation:

$$
\begin{gathered}
\frac{[\mathrm{LThDP}]}{[\mathrm{ThDP}]}=\frac{k_{2}^{\prime}}{k_{3}^{\prime}}=a \\
\frac{[\mathrm{HEThDP}]}{[\mathrm{LThDP}]}=\frac{k_{3}^{\prime}}{k_{4}^{\prime}}=b
\end{gathered}
$$

Combining eqs 3,7 , and 8 will give

$$
\begin{aligned}
& k_{2}^{\prime}=k_{\mathrm{obs}}(1+a+a b) \\
& k_{3}^{\prime}=k_{\mathrm{obs}} \frac{(1+a+a b)}{a} \\
& k_{4}^{\prime}=k_{\mathrm{obs}} \frac{(1+a+a b)}{a b}
\end{aligned}
$$

The rate constant of reoxidation $k_{5}^{\prime}$ has been determined separately by kinetic analysis of the oxidative half-reaction using stopped-flow kinetics (12).

The net rate constant of the phosphorolytic cleavage of AcThDP $\left(k_{6}^{\prime}\right)$ can be obtained as follows. If AcThDP will be isolated at steady state under aerobic conditions, its relative concentration compared to that of any other ThDP intermediate (ThDP, LThDP, and HEThDP) gives a relative measure of $k_{6}^{\prime}$ compared to the rate constant of the breakdown of this intermediate $\left(k_{2}^{\prime}, k_{3}^{\prime}\right.$, and $\left.k_{4}^{\prime}\right)$. Because all of these rate constants have been already determined, the calculation of $k_{6}^{\prime}$ is straightforward.

$$
\frac{[\mathrm{AcThDP}]}{[\mathrm{ThDP}]}=\frac{k_{2}^{\prime}}{k_{6}^{\prime}} \frac{[\mathrm{AcThDP}]}{[\mathrm{LThDP}]}=\frac{k_{3}^{\prime}}{k_{6}^{\prime}} \frac{[\mathrm{AcThDP}]}{[\mathrm{HEThDP}]}=\frac{k_{4}^{\prime}}{k_{6}^{\prime}}
$$

It should be noted that AcThDP is in equilibrium with its internal hydrate and a carbinolamine form under the chosen conditions (3) and that all three species occur in comparable fractions. It is therefore challenging to observe AcThDP in only small amounts being present at steady state because the analytical signal splits off further. High active-site concentrations of the protein are needed to allow detection of small amounts of AcThDP by ${ }^{1} \mathrm{H}$ NMR.

As a test when all substrates are saturating, $k_{6}^{\prime}$ can be calculated from the relationship of $k_{\text {cat }}$ and the net rate constants of the catalytic sequence according to eq 13 .

$$
\frac{1}{k_{\mathrm{cat}}}=\frac{1}{k_{2}^{\prime}}+\frac{1}{k_{3}^{\prime}}+\frac{1}{k_{4}^{\prime}}+\frac{1}{k_{5}^{\prime}}+\frac{1}{k_{6}^{\prime}}
$$

If the kinetic treatment is correct, both methods should give the same $k_{6}^{\prime}$ values.

We want to point out that the kinetic NMR analysis refers to forward catalysis. Some of the steps are certainly reversible, for instance, step 2 that is governed by $k_{2}^{\prime}$. The net rate constant $k_{2}^{\prime}$ is related to microscopic rate constants as follows:

$$
k_{2}^{\prime}=\frac{k_{2} k_{3}}{k_{-2}+k_{3}}
$$

However, we know from earlier studies that the forward commitment factor of LThDP decomposition $\left(k_{3} / k_{-2}\right)$ in LpPOX ranges between 5 and 6 (12). Thus, $k_{2}^{\prime} \approx k_{2}$. All other steps of catalysis can be also kinetically referred to as forward-directed catalysis substrate saturation provided.

Determination of the Redox Potentials of FAD in LPPOX. To determine the redox potential of the enzyme-bound FAD in $L p P O X$, the enzyme was reductively titrated under anaerobic conditions with sodium dithionite in the presence and absence of redox dyes with known potential. Indigo disulfonate $\left(E_{\mathrm{m}}=-65 \mathrm{mV}\right.$ at $\left.\mathrm{pH} 6.0\right)$, cresyl violet $\left(E_{\mathrm{m}}=\right.$ $-93 \mathrm{mV}$ at $\mathrm{pH} 6.0)$, and phenosafranine $\left(E_{\mathrm{m}}=-208 \mathrm{mV}\right.$ at $\mathrm{pH}$ 6.0) were employed for analysis (20). Because no reference for the midpoint potential of cresyl violet at $\mathrm{pH} 6$ is available in the literature, it has been determined by simultaneous reductive titration with indigo disulfonate in a parallel experiment.

In a typical experiment with the enzyme, $1 \mathrm{~mL}$ of $15 \mu \mathrm{M}$ $L p \mathrm{POX}$ (active-site concentration) was placed in an anaerobic cuvette in either $0.2 \mathrm{M}$ potassium phosphate at $\mathrm{pH} 6.0$ or 
$0.1 \mathrm{M} \mathrm{Mes} / \mathrm{NaOH}$ at $\mathrm{pH} 6.0$ containing $1 \mu \mathrm{M}$ benzyl viologen for rapid redox equilibration. Experiments were carried out both in the absence of redox dyes to determine the thermodynamic stabilization of the FAD semiquinone $\left(\mathrm{FAD}_{\mathrm{sq}}\right)$ and in the presence of $10-20 \mu \mathrm{M}$ redox dye of known potential for the estimation of the midpoint potential of the enzyme-bound flavin. Anaerobiosis was established by repeated cycles of alternate evacuation and flushing with oxygen-free nitrogen. For the whole duration of the procedure, the enzyme was kept on ice to prevent aggregation. The enzyme was then reduced by stepwise and careful addition of $0.2-0.5 \mu \mathrm{L}$ aliquots of an anaerobic sodium dithionite solution at $15^{\circ} \mathrm{C}$. Sodium dithionite was freshly made prior to every experiment, and its concentration was adjusted after reductive titration of free FAD. UV-vis absorbance spectra of the enzyme solution were recorded from 300 to $700 \mathrm{~nm}$ before and during the reductive titration using an Uvikon Kontron spectrophotometer. Sufficient time was given to allow redox equilibration in the anaerobic cuvette. To ensure that this was accomplished, the kinetics of reduction was followed upon single additions of the reductant.

The thermodynamic separation of the two single-electron transfers (the $\Delta E$ between the $\mathrm{FAD}_{\mathrm{ox}} / \mathrm{FAD}_{\mathrm{sq}}$ and $\mathrm{FAD}_{\mathrm{sq}}$ / $\mathrm{FAD}_{\text {red }}$ couples) was estimated from the maximal percentage of semiquinone formation during reduction in the absence of a dye (20)

$$
\begin{gathered}
\Delta E=59 \mathrm{mV} \log K \\
K=\frac{\left[\mathrm{FAD}_{\mathrm{sq}}\right]^{2}}{\left[\mathrm{FAD}_{\mathrm{red}}\right]\left[\mathrm{FAD}_{\mathrm{ox}}\right]}
\end{gathered}
$$

The extent of semiquinone formation was estimated both graphically by plotting the changes in absorbance at the maximum wavelength of the semiquinone $(590 \mathrm{~nm})$ versus that of the oxidized form (407 nm) and by "Eigenfactor" analysis of complete UV-vis spectra using Specfit32 (21).

The midpoint potential of the enzyme-bound flavin was determined in the presence of a dye with a redox potential $\pm 30 \mathrm{mV}$ to that of the enzyme-bound FAD. The concentrations of the oxidized and reduced dye forms and those of the different flavin species were determined from the absorbance values at various wavelengths where (i) the contribution of either the enzyme or that of the dye are negligible or (ii) by using isosbestic points of the different enzyme and reference dye forms. The calculated concentrations of oxidized and reduced dye were then transformed into the redox potential of the system at equilibrium according to the Nernst equation

$$
E=E_{\mathrm{m}}+\frac{2.3 R T}{n F} \lg \left(\frac{\left[\mathrm{dye}_{\mathrm{ox}}\right]}{\left[\text { dye }_{\mathrm{red}}\right]}\right)
$$

where $R$ is the gas constant, $T$ is the absolute temperature, $n$ the number of electrons transferred, and $F$ is Faraday's constant $\left(96.5 \mathrm{~kJ} \mathrm{~V}^{-1} \mathrm{~mol}^{-1}\right)$. The course of the absorbance $A$ of the enzyme-bound flavin was directly plotted and fitted to eq 18, which can be derived from a combination of Lambert-Beer's law and the Nernst equation for a twoelectron process

$$
A=\frac{a+b 10^{\left(E_{\mathrm{x}}-E\right) / 003}}{1+10^{\left(E_{\mathrm{x}}-E\right) / 003}}
$$

where $a$ represents the absorbance of the oxidized enzyme, $b$ represents that of the reduced enzyme, $E_{\mathrm{x}}$ is the midpoint potential of the flavin, and $E$ is the potential of the system. The individual potentials of the $\mathrm{FAD}_{\mathrm{ox}} / \mathrm{FAD}_{\mathrm{sq}}\left(E_{1}\right)$ and $\mathrm{FAD}_{\mathrm{sq}} / \mathrm{FAD}_{\text {red }}\left(E_{2}\right)$ couples were then calculated from the midpoint potential and the determined $\Delta E$ assuming an equidistant separation of $E_{1}$ and $E_{2}$ from the midpoint potential.

Fluorescence Resonance Energy Transfer (FRET) Studies. In contrast to ThDP that is nonfluorescent at room temperature, the coenzymatically inactive analogue N3'-pyridylThDP emits with a $\lambda_{\max } \approx 370 \mathrm{~nm}$. The FAD in LpPOX absorbs at this wavelength, thus opening up the possibility for FRET between excited N3'-pyridyl-ThDP and FAD and in a further instance to use this FRET as a sensitive molecular probe for alterations of the intercofactor distance.

Apo- $L p$ POX (1.5 mg/mL) was incubated with $1 \mathrm{mM} \mathrm{N3}$ pyridyl-ThDP (inset in Figure 8) in the presence of $1 \mathrm{mM}$ $\mathrm{Mn}^{2+}$ and $100 \mu \mathrm{M}$ FAD in $0.2 \mathrm{M}$ potassium phosphate buffer containing $10 \%(\mathrm{v} / \mathrm{v})$ glycerol overnight at $6{ }^{\circ} \mathrm{C}$. Thereafter, excess cofactors and $\mathrm{Mn}^{2+}$ were removed by gel filtration using a HiTrap G-25 desalting column (Pharmacia Amersham). Fluorescence spectra of $0.62 \mathrm{mg} / \mathrm{mL}$ holo-(N3'pyridyl-ThDP-FAD-Mn $\left.{ }^{2+}\right)-L p P O X$ in either $0.2 \mathrm{M}$ potassium phosphate buffer at $\mathrm{pH} 6.0$ or $0.1 \mathrm{M} \mathrm{Mes} / \mathrm{NaOH}$ at $\mathrm{pH} 6.0$ were recorded at $15{ }^{\circ} \mathrm{C}$ using a Fluoromax fluorescence spectrometer (slit width $=2 \mathrm{~nm}$ for excitation and emission). With a $\lambda_{\text {exc }}$ of $315 \mathrm{~nm}, 10$ individual spectra (integration time of $0.2 \mathrm{~s}$ ) were averaged for analysis. Emission spectra of enzyme-bound FAD were recorded from 320 to $600 \mathrm{~nm}$. When wild-type $L p P O X$ (with native ThDP) is irradiated at this wavelength under identical experimental conditions as described above, no significant emission of the enzymebound FAD is detectable (data not shown). The enzymebound $\mathrm{Mn}^{2+}$ in POX does not significantly quench the fluorescence of the thiamin and flavin cofactors because similar fluorescence intensities were observed when $\mathrm{Mg}^{2+}$ was used instead of $\mathrm{Mn}^{2+}$.

\section{RESULTS}

Steady-State Kinetics of LpPOX in the Presence and Absence of Phosphate. Former studies have indicated that the catalytic efficiency of $L p P O X$ is strictly dependent on the presence of the substrate phosphate (2), which is thought to be engaged in the phosphorolytic cleavage of the putative AcThDP intermediate (compare to Scheme 1). When phosphate is absent, however, the enzyme still turns over pyruvate and oxygen, although with a significantly reduced $k_{\text {cat }}$. We have concluded that the solvent/hydroxide ion may replace phosphate in the final reaction step and eventually trap the acetyl moiety of AcThDP, yielding acetate (12). As shown in Figure 2, the total consumption of the final electronacceptor oxygen in the solution by the enzyme monitored by stopped-flow kinetics occurs within $2 \mathrm{~s}$ in phosphate buffer, whereas under the same conditions but in phosphatefree buffer, it is completed only after approximately $60 \mathrm{~s}$. Analysis of the stopped-flow progress curves according to Gibson et al. (13) yields specific activities of 0.3 unit/mg 

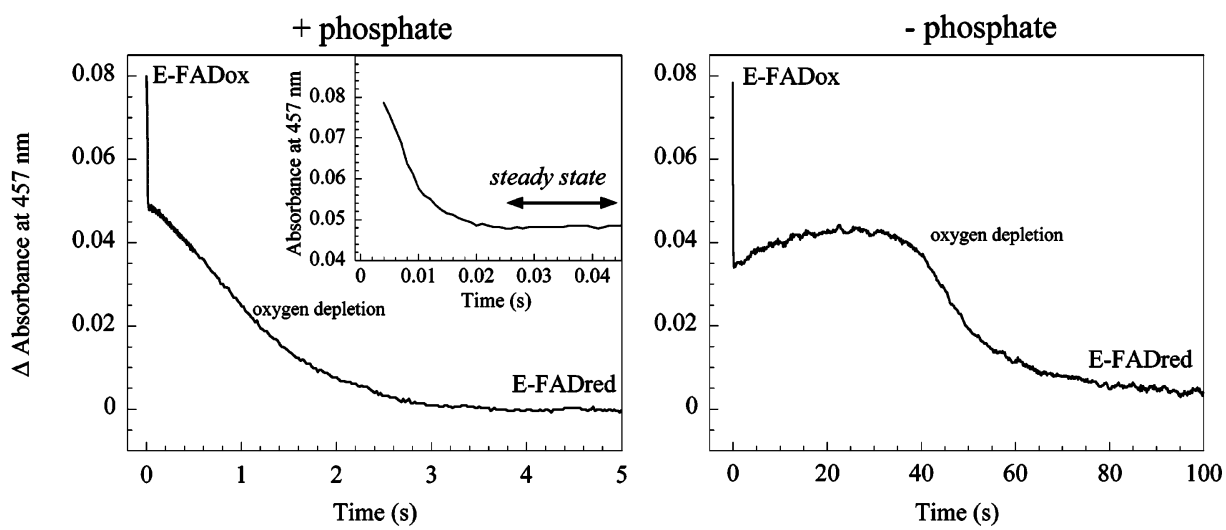

FIGURE 2: Effect of phosphate on the reaction of $L p P O X$ with pyruvate in the presence of oxygen. (Left panel) Time course of the absorption monitored at $457 \mathrm{~nm}$ for the reaction of $16 \mu \mathrm{M} \mathrm{LpPOX}$ with $50 \mathrm{mM}$ pyruvate and in the presence of phosphate $(0.2 \mathrm{M}$ potassium phosphate buffer at pH 6.0). (Right panel) Same conditions, however, in $0.1 \mathrm{M} \mathrm{Mes} / \mathrm{NaOH}$. The reaction was conducted at $25^{\circ} \mathrm{C}$ in the stopped-flow instrument. (Inset) Initial phase of the reaction depicted in the main panel and indicating the steady state.
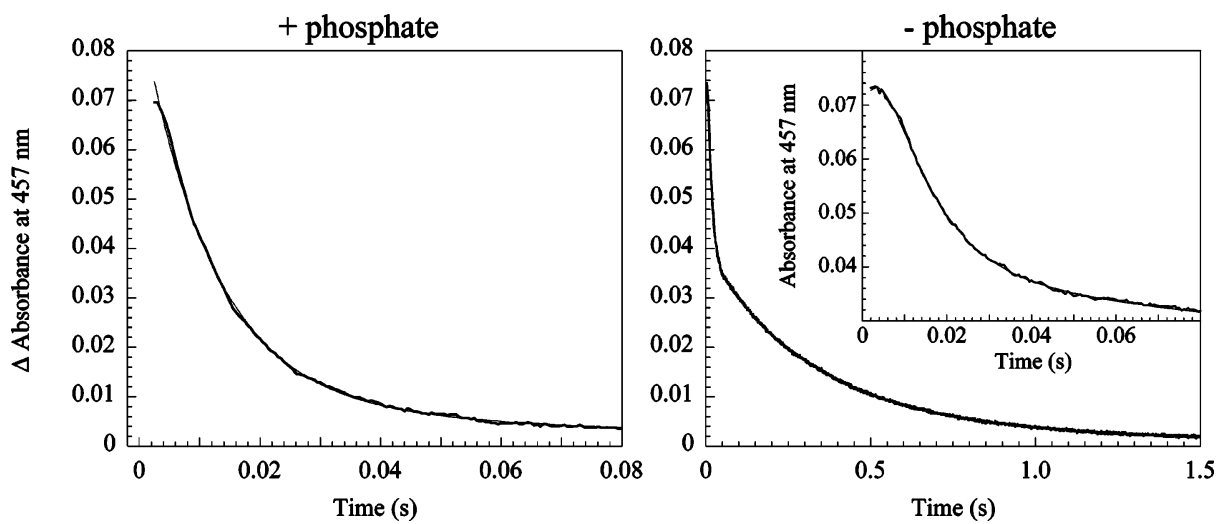

FIGURE 3: Effect of phosphate on the reaction of $L p \mathrm{POX}$ with pyruvate in the absence of oxygen. A solution of $15 \mu \mathrm{M} L p \mathrm{POX}$ in $0.2 \mathrm{M}$ potassium phosphate buffer at $\mathrm{pH} 6.0$ (left panel) or $0.1 \mathrm{M} \mathrm{Mes} / \mathrm{NaOH}$ at $\mathrm{pH} 6.0$ (right panel) was reacted with $50 \mathrm{mM}$ pyruvate at $25^{\circ} \mathrm{C}$ under anaerobic conditions in the stopped-flow instrument. Kinetic traces were analyzed using a single- or double-exponential function. Fitted curves are shown as solid lines and yielded rate constants of $k=78 \pm 1 \mathrm{~s}^{-1}$ (left panel) and $k_{1}=81 \pm 1$ and $k_{2}=2.9 \pm 0.1 \mathrm{~s}^{-1}$ (right panel). (Inset in the right panel) Initial phase of the reaction in the absence of phosphate.

$\left(k_{\text {cat }}=0.32 \mathrm{~s}^{-1} /\right.$ active site $)$ in phosphate-free medium and 32 units/mg ( $k_{\mathrm{cat}}=35 \mathrm{~s}^{-1}$, extrapolated for saturating concentrations of all substrates) in phosphate buffer. A study of the steady-state parameters of $L p P O X$ catalysis employing DCPIP as an artificial electron acceptor and monitoring the oxygen consumption directly with an oxygen electrode yields comparable specific activities as with the stopped-flow/ Gibson method (data not shown). Model studies validated that DCPIP could trap the enamine formed upon decarboxylation of LThDP sufficiently fast to not allow the enamine intermediate to accumulate (22).

Reductive Half-Reaction of LpPOX in the Presence and Absence of Phosphate. It was no surprise that turnover of $L p \mathrm{POX}$ is significantly affected by the presence of phosphate (see above). Besides this expected impact on the overall catalysis, we have detected a very peculiar dependence of the reductive half-reaction (steps 1-4 in Scheme 1) on phosphate, which is thought to play a role only in later steps of catalysis. Under anaerobic conditions, the enzyme-bound flavin in $L p P O X$ is reduced with a maximum apparent firstorder rate constant of $78 \pm 1 \mathrm{~s}^{-1}$ at $25^{\circ} \mathrm{C}$ when pyruvate and phosphate are saturating. Under the same conditions but with phosphate absent, the reduction is clearly biphasic when monitored at $457 \mathrm{~nm}$ (intrinsic FAD absorbance), with apparent rate constants of $81 \pm 1 \mathrm{~s}^{-1}$ for the first phase and $2.9 \pm 0.1 \mathrm{~s}^{-1}$ for the second phase (Figure 3). Apparently, the first phase proceeds with a rate comparable to that observed in the presence of phosphate. Therefore, we conclude that (i) phosphate does specifically enhance the rate of a catalytic step that kinetically determines the slow, second reduction phase and (ii) phosphate has no or only a marginal catalytic role on all preceding steps of catalysis.

Detection of Reaction Intermediates by Time-Resolved Spectroscopy. We have reported recently that flavin radicals are not detectable in the pre-steady state and steady state of $L p \mathrm{POX}$ catalysis when the enzyme is reacted with pyruvate, phosphate, and oxygen (12). In an extension to these studies, we have analyzed the time-resolved absorbance spectra of $L p \mathrm{POX}$ in the course of the reductive half-reaction in the presence and absence of phosphate. No transient flavin radicals are detectable in phosphate buffer (Figure 4). This is compatible with the transfer of the first reducing equivalent being significantly slower than that of the second. In contrast, there is striking evidence for transient formation of FAD radicals when $L p \mathrm{POX}$ is reacted with pyruvate in phosphatefree buffer (parts A and B of Figure 5). In a first phase, which kinetically corresponds to the fast one observed in the single-wavelength experiments at $457 \mathrm{~nm}$, an increase in absorbance is observable in the spectral region $\lambda>500$ $\mathrm{nm}$. Moreover, there is a significant shift of the $\lambda_{\max }$ in the $350-400 \mathrm{~nm}$ region. In the second phase, the absorbance at $\lambda>500 \mathrm{~nm}$ disappears and the two-electron reduced form 


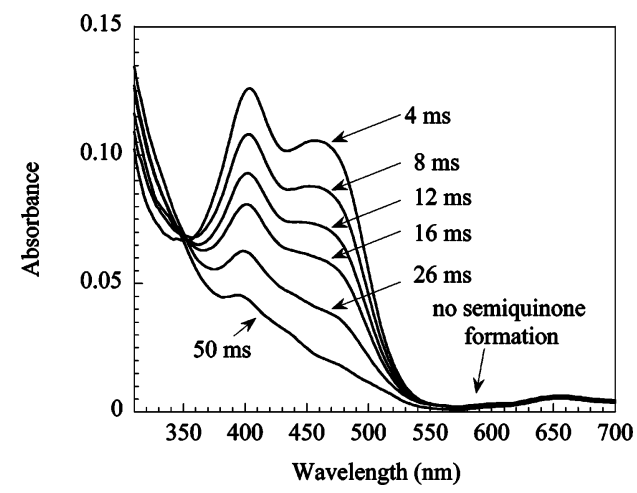

FIGURE 4: Spectral course of the LpPOX reductive half-reaction in the presence of pyruvate and phosphate. A solution of $13 \mu \mathrm{M}$ $L p \mathrm{POX}$ (active-site concentration) was reacted with $50 \mathrm{mM}$ pyruvate in 0.2 potassium phosphate buffer at $\mathrm{pH} 6.0$ and $25^{\circ} \mathrm{C}$ under anaerobic conditions. The spectra shown are a selection and were recorded at $4,8,12,16,26$, and $50 \mathrm{~ms}$ upon mixing. All spectra are corrected for buffer and substrate contributions. The last spectrum corresponds for practical purposes to the end of the reaction. Note the absence of absorbance in the 550-700 $\mathrm{nm}$ region that would correspond to formation of flavin semiquinone(s). Compare to Figure 5.

of the flavin is formed. A further kinetic analysis of the reductive half-reaction monitored at $550 \mathrm{~nm}$ (Figure 5C) verifies that buildup and decay of the transient FAD species corresponds kinetically to the fast and slow phases of FAD reduction monitored at $457 \mathrm{~nm}$. The deconvolution of the obtained spectra with Specfit32 yields a spectroscopic species that is likely composed of a mixture of the blue and red FAD semiquinones. The calculated spectrum of the intermediate species is closely similar to that obtained by deconvolution of spectra recorded in the course of reductive titration of $L p \mathrm{POX}$ in the absence of a dye (compare Figure 7B). It thus appears that in the absence of phosphate one electron is transferred rapidly $\left(k_{\mathrm{app}}=81 \mathrm{~s}^{-1}\right)$ from the HEThDP enamine to the flavin. The resulting HEThDP radical/FAD semiquinone pair then slowly decomposes $\left(k_{\text {app }}=3 \mathrm{~s}^{-1}\right)$ to form fully reduced FAD and AcThDP. In the presence of phosphate, the lifetime of the HEThDP/FAD radical pair is decreased at least $10^{2}-10^{3}$ fold if not more.

Analysis of the Temperature Dependence of the Observed Rate Constant of FAD Reduction According to the Marcus Theory and the Transition-State Theory. The observation of a transient $\mathrm{HEThDP} / \mathrm{FAD}_{\mathrm{sq}}$ radical pair raises the possibility that the different kinetic phases seen in the stopped-flow experiments may reflect true ET events. As it has been briefly outlined in the Materials and Methods, an analysis of the temperature dependence of the apparent rate constants of this process can be used to distinguish between chemically gated and true ET reactions. To this purpose, we determined the apparent rate constants of FAD reduction at different temperatures both in the presence and absence of phosphate. The experimental data were fitted to eqs 4 and 5 (parts A and B of Figure 6 and Table 1). The first-order rate constants of FAD reduction in the presence of phosphate show a similar temperature dependence as those of the first, fast phase in phosphate-free buffer. Fitting these data gave only a poor fit and yielded an $H_{\mathrm{AB}}$ of $\approx 120 \mathrm{~cm}^{-1}$, suggesting that ET from the HEThDP enamine to FAD is at least partially gated by preceding adiabatic steps of catalysis, i.e., formation of LThDP and decarboxylation of the latter (compare Scheme 1). It was also impossible to fit these data by means of the transition-state theory using eq 6 , because a nonlinear dependence is observed in the Eyring plot (inset in Figure 6A and Table 1). The plot rather suggests a change in the rate-determining step(s) with increasing temperature.

In contrast, the slow decay of the radical pair, which is observed exclusively in phosphate-free medium, appears to reflect a true ET reaction because the obtained coupling constant $H_{\mathrm{AB}}\left(16 \mathrm{~cm}^{-1}\right)$ is well below the limit of an adiabatic reaction $\left(80 \mathrm{~cm}^{-1}\right)$. This rather high value is probably due to the small edge-edge intercofactor distance of only $7 \AA$, allowing for strong coupling. A fitting to eq 5 using the already determined $\Delta G^{\circ}(-38.6 \mathrm{~kJ} / \mathrm{mol})$ and $\lambda(1.9 \mathrm{eV})$ yields an electronic decay factor $\beta$ of approximately $2 \AA^{-1}$ and a donor-acceptor distance $r$ of $9 \AA$. Given that theoretical and experimental studies suggest $\beta$ to range between 0.7 and $1.4 \AA^{-1}$ in most proteins $(15-18)$, the calculated value appears somewhat high. On the other hand, an inspection of the X-ray crystallographic structure of LpPOX shows that neither active-site side chains nor the backbone are directly located between the two cofactors. Because there is an evident lack of orbitals in the putative direct edge-edge pathway, a large decay factor seems to be reasonable. Fitting the data to eq 5 with a fixed $\beta$ of 1.4 yields a donor-acceptor distance of $15 \AA$, which is clearly larger than the observed edge-edge distance of $7 \AA$ in the $X$-ray structure. On the other hand, the linear ET path distance between the thiazolium ring of ThDP and the redox active pyrimidine/pyrazine part of the FAD isoalloxazine part is some $11-13 \AA$, and the effective ET path length might be even longer if the electrons do not tunnel directly but also use orbitals of proximate side chains such as Phe 479 or Phe121 (Figure 1). Although there might be some uncertainty regarding $\beta$, we consider the disappearance of the radical species to correspond to a true ET reaction because (i) all preceding chemical (adiabatic) steps and transfer of the first reducing equivalent are considerably faster and thus do not gate the radical decay, (ii) a coupling to a preceding equilibrium as discussed for interprotein ET reactions appears to be unreasonable for the intramolecular ET in $L p P O X$, and (iii) $H_{\mathrm{AB}}$ is well below the nonadiabatic limit of an ET reaction.

Intermediate Analysis by Acid-Quench/l H NMR Spectroscopy and Calculation of the Unimolecular Net Rate Constants. We have analyzed the occurrence of covalent ThDP intermediates in $L p$ POX catalysis upon defined reaction times ranging from $5 \mathrm{~ms}$ to $5 \mathrm{~s}$ (Figure $\mathrm{S} 1$ in the Supporting Information). Similar to what was observed in the stoppedflow studies (see Figure 2A), the true steady state is limited to an extremely short time period from 22 to $45 \mathrm{~ms}$ when all substrates are present. After the steady state, the ongoing depletion of the substrate oxygen leads to the exclusive accumulation of the HEThDP enamine (data not shown). Under true steady-state conditions, we observe a large fraction of the tetrahedral substrate-ThDP adduct LThDP and minor fractions of HEThDP and ThDP (Figure S1 in the Supporting Information). Calculation of the individual net rate constants according to eqs $7-12$ shows that the decarboxylation reaction is the slowest in the reductive halfreaction sequence, whereas carbonyl addition of pyruvate to ThDP and ET are slightly faster (Scheme 3). The calculated rate constants demonstrate that ET in the course of FAD reduction is indeed kinetically gated by preceding 

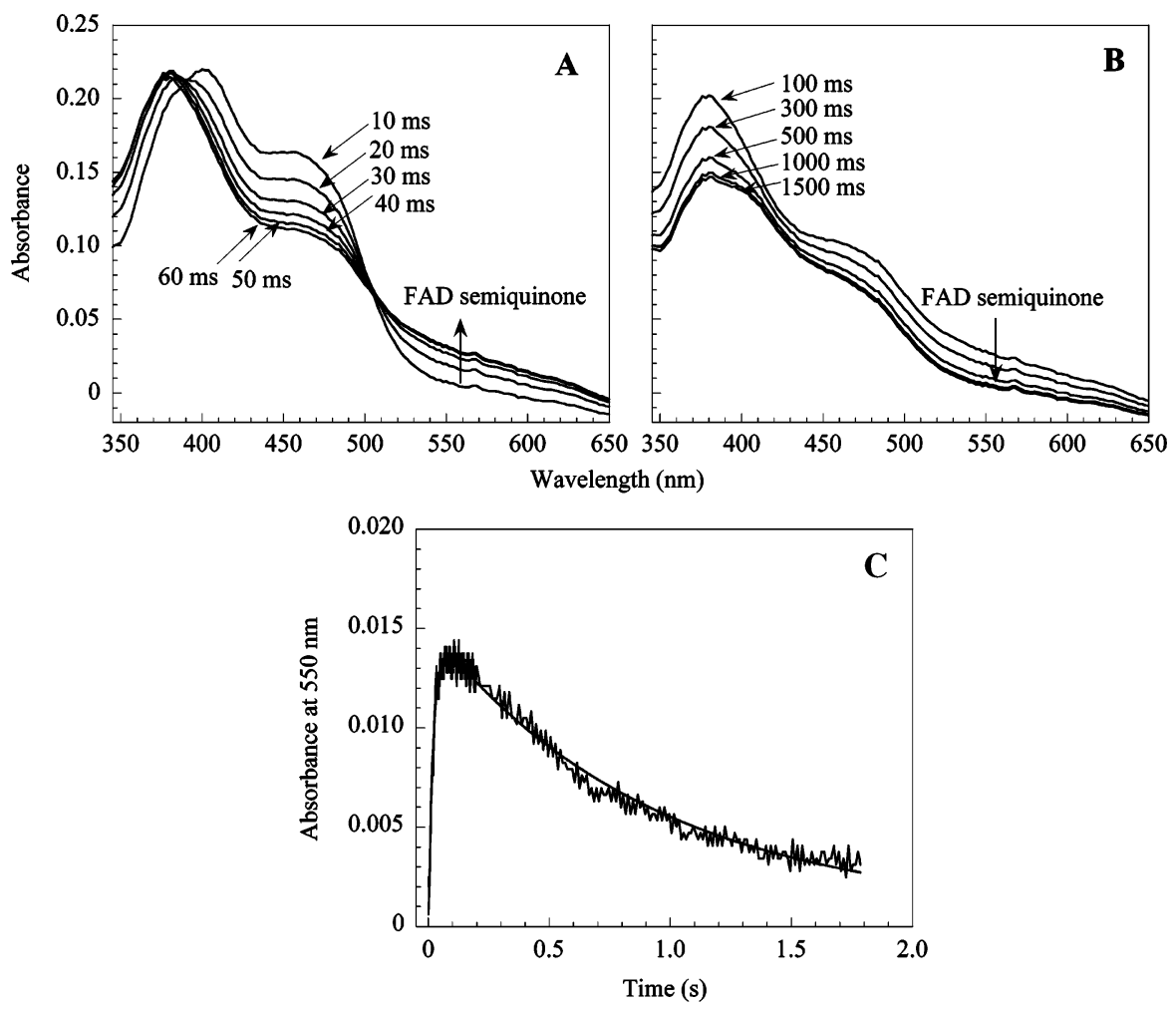

FIGURE 5: Spectral course of the $L p P O X$ reductive half-reaction in the presence of pyruvate and absence of phosphate. Conditions are the same as detailed in the caption of Figure 4, however, using $19 \mu \mathrm{M} L p \mathrm{POX}$ and in $0.1 \mathrm{M}$ Mes/NaOH buffer at pH 6.0. (A and B) Selected spectra representing the changes occurring in two separate, subsequent phases. (C) Progress curve of the reaction $(L p \mathrm{POX}=16 \mu \mathrm{M})$ monitored at $550 \mathrm{~nm}$, the absorbance of the neutral, blue FAD semiquinone. The solid line is the fit obtained using a double-exponential equation.

steps of catalysis, thus supporting the results of the Marcus analysis.

When pyruvate and phosphate are saturating, we did not observe AcThDP at steady state (Figure S1 in the Supporting Information). This is intriguing and implies that either phosphorolysis of AcThDP is extremely fast compared to all other steps of catalysis or, alternatively, AcThDP is not on the major pathway.

In the absence of phosphate, hydrolysis of AcThDP is the slowest step of this futile catalytic cycle as evidenced by the accumulation of AcThDP at steady state (Figure S1 in the Supporting Information). The small fraction of unsubstituted ThDP results from the slow hydrolysis of AcThDP after acid-quench isolation in the course of NMR data acquisition.

Determination of the Redox Potentials of FAD in LPPOX. The reductive titration of $L p P O X$ by sodium dithionite in the absence of a redox dye leads to the formation of an oneelectron reduced (semiquinone) flavin intermediate. Both graphical analysis and deconvolution of the obtained spectra by Eigenfactor analysis suggest a thermodynamic stabilization of the $\mathrm{FAD}_{\mathrm{sq}}$ to a $55-57 \%$ extent. This corresponds to a separation of the $\mathrm{FAD}_{\mathrm{ox}} / \mathrm{FAD}_{\mathrm{sq}}$ and $\mathrm{FAD}_{\mathrm{sq}} / \mathrm{FAD}_{\text {red }}$ potentials by approximately $50-55 \mathrm{mV}$ (Figure 7). The thermodynamic stabilization of FAD radicals is independent of phosphate. As already stated in the section describing the rapid kinetics, the FAD semiquinone appears to be composed of both the anionic (red) and neutral (blue) forms.

The midpoint potential of the enzyme-bound FAD in $L p \mathrm{POX}$ was also determined in the presence and absence of phosphate using the dye equilibration method (Figure S2 in the Supporting Information). Among the different dyes tested, indigo disulfonate turned out to be the most suitable one for the determination of the redox potential of FAD in $L p$ POX. The estimated midpoint potential of FAD in $\operatorname{LpPOX}\left(E_{\mathrm{m}}=\right.$ $-62 \mathrm{mV})$ is almost the same as that of the dye $(E=-65$ $\mathrm{mV})$. Most notably, the midpoint potential of FAD is identical in the presence and absence of phosphate. This result rules out phosphate as a modulator of the ET reaction through an effect on the flavin redox potential.

FRET Studies. In view of the tremendous impact of phosphate on the rate of intercofactor ET, a possible structural role of phosphate ought to be discussed. If phosphate were to induce a conformational change upon binding to the active site, it might affect the intercofactor distance between the flavin and ThDP and thus ultimately influence the ET rate. This hypothesis was tested by FRET studies. For this, we replaced the nonfluorescent ThDP by the fluorescent but coenzymatically inactive analogue N3'pyridyl-ThDP (scheme in Figure 8A). The fluorescence emission of free $\mathrm{N}^{\prime}$-pyridyl-ThDP overlaps in part with the flavin absorbance in $L p P O X$ (Figure 8A). In the case of FRET, the FAD emission would serve as a sensitive molecular probe. As a control, it was established that upon excitation of the native cofactor ThDP at $315 \mathrm{~nm}$ there is no emission because of the flavin chromophore (data not shown).

Selective excitation of the enzyme reconstituted with N3'pyridyl-ThDP at $315 \mathrm{~nm}$ leads to a weak flavin fluorescence emission maximal at $530 \mathrm{~nm}$. From this, we conclude that FRET between the two cofactors does occur. It must be noted that the emission because of FRET is less than 5\% of that 

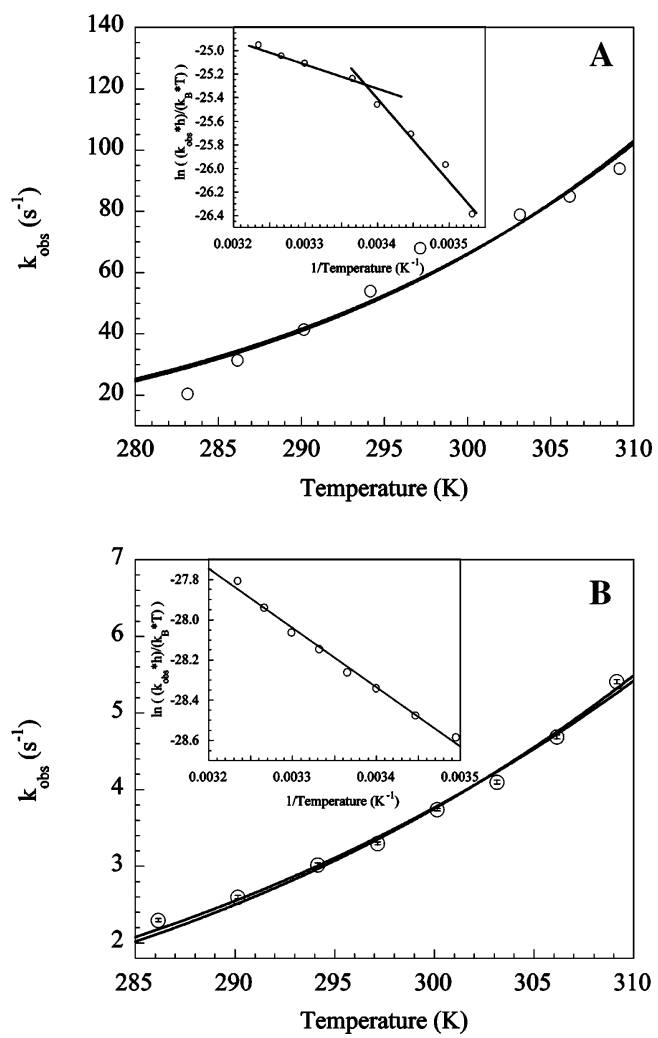

FIGURE 6: Temperature dependence of the apparent rate constants of FAD reduction analyzed by the Marcus theory and the Eyring transition-state theory (insets). (A) Analysis of the reduction of LpPOX with pyruvate in the presence of phosphate. A similar result has been obtained for the first fast phase of FAD reduction in the absence of phosphate. (B) Analysis of the slow phase (radical decay) of the reduction of $L p P O X$ with pyruvate in the absence of phosphate.

observed after specific excitation of the flavin at $407 \mathrm{~nm}$ (absorbance maximum). This poor sensitivity is mainly the result of the low quantum yield of N3'-pyridyl-ThDP fluorescence. Under the conditions used $(0.1 \mathrm{M}$ potassium phosphate buffer at $\mathrm{pH} 6.0$ and $25{ }^{\circ} \mathrm{C}$ ), we estimated the quantum yield of the free analogue to be 0.0112 .

Qualitatively, phosphate appears to exhibit no detectable structural effect on the enzyme in terms of changing the distance between both cofactors because nearly similar FRET efficiencies were found in the absence and presence of phosphate (Figure 8B). In fact, in the absence of phosphate, the transfer efficiency is even some 5-10\% larger than in its presence. Therefore, structural changes in terms of a putative annealing of both cofactors caused by binding of phosphate cannot account for the kinetic effects of phosphate on the ET reaction in $L p P O X$.

Does Phosphate Have a Kinetic Effect on ET of Related ThDP-Dependent Flavoenzymes? In addition to LpPOX, the related POX from E. coli $(E c \mathrm{POX})$ and acetohydroxyacid synthase from E. coli (EcAHAS II) were also shown to catalyze an ET from the ThDP enamine-intermediate to the flavin cofactor $(10,23)$. In contrast to $L p P O X$, the elusive AcThDP intermediate formed upon oxidation of the enamine is not subject to phosphorolytic cleavage in these two enzymes but rather undergoes hydrolysis to yield acetate. In view of the observed effect of phosphate on the ET rate in $L p \mathrm{POX}$, we aimed to characterize a potential role of phosphate for the related reactions catalyzed by $E c P O X$ and
AHAS. In both enzymes, the observed rate constant of ET is independent of phosphate (data not shown). The unique effect of phosphate for ET in $L p$ POX catalysis thus suggests that the intramolecular redox reaction is intimately connected with the formation of acetyl phosphate, which, in turn, is exclusively catalyzed by $L p$ POX.

\section{DISCUSSION}

The single-step analysis of $L p \mathrm{POX}$ catalysis has evidenced that phosphate is unexpectedly not only involved in the phosphorolysis of the putative AcThDP intermediate formed upon oxidation of the HEThDP enamine but also plays a crucial role for the intramolecular redox reaction between the thiamin enamine intermediate and the flavin cofactor. The kinetic examination of the reductive half-reaction, which encompasses all catalytic steps up to and including the reduction of the flavin by the enamine (steps 1-4 in Scheme 1 ), revealed that the FAD is fully reduced in a fast monophasic process in the presence of phosphate. No transient flavin radicals are kinetically stabilized. This requires that the decay of a putative intermediate HEThDP/ $\mathrm{FAD}_{\mathrm{sq}}$ radical pair to be markedly faster than its formation. The inability to observe radical intermediates suggests the radical decay to occur at least 1 order of magnitude faster than its build-up $\left(k_{\text {decay }}>800 \mathrm{~s}^{-1}\right)$.

In contrast to this, when $L p P O X$ is reacted with pyruvate in the absence of phosphate, reduction of the flavin occurs in two phases with the HEThDP radical/FAD ${ }_{\text {sq }}$ pair being an observable intermediate. In a fast phase $\left(k_{\text {app }}=81 \mathrm{~s}^{-1}\right)$, the first of the two reducing equivalents is transferred from the enamine to the flavin, yielding the radical pair, which, in a further slow phase corresponding to a true ET reaction, decays $\left(k_{\text {app }}=3 \mathrm{~s}^{-1}\right)$ to yield the fully reduced flavin and AcThDP. We conclude that phosphate has no catalytic role for all steps that precede the transfer of the second reducing equivalent. Apparently, phosphate exclusively decreases the lifetime of the HEThDP radical by several orders of magnitude. What is the mechanism of this effect? In theory, there are several possibilities that might explain this phenomenon.

According to the Marcus theory, the increase of the rate of an ET reaction may be caused (14) by (i) a larger intrinsic driving force of the reaction through an increase in the difference of the redox potentials of the donor/acceptor pair, (ii) a decrease of the distance between the donor and acceptor, (iii) lowering the reorganization energy $\lambda$, (iv) lowering the decay factor $\beta$ by providing orbitals for an optimal ET path, or (v) modification of the chemistry of the reaction. On the basis of our kinetic, thermodynamic, and structural studies, the feasibility of some mode will be discussed.

The FRET experiments argue against structural changes induced by phosphate. Even more convincing and further buttressing the FRET data, we recently succeeded in trapping intermediates in the active site in the absence and presence of phosphate (manuscript in preparation) with nearly identical spatial orientations of ThDP and FAD in the X-ray structure. Thus, a change of the ET path length can be ruled out.

Another attractive explanation is the so-called wire mechanism. It may be assumed that phosphate binds in the activesite cavity in a way that it could lend its orbitals for an 
Table 1: Parameters Obtained from Fitting the Apparent Rate Constants of FAD Reduction at Different Temperatures According to the Marcus Theory (eqs 4 and 5) and the Eyring Theory (eq 6)

\begin{tabular}{|c|c|c|}
\hline & $\begin{array}{l}\text { slow radical decay in } \\
\text { the absence of phosphate }\end{array}$ & $\begin{array}{l}\text { reduction in the presence of phosphate } \\
\text { and first fast reduction phase in } \\
\text { the absence of phosphate }\end{array}$ \\
\hline$H_{\mathrm{AB}}\left(\mathrm{cm}^{-1}\right)$ & $16 \pm 5^{a}$ & {$[120]^{b}$} \\
\hline$\lambda(\mathrm{eV})$ & $1.9 \pm 0.2$ & {$[1.8]$} \\
\hline$\Delta G^{\circ}\left(\mathrm{kJ} \mathrm{mol}^{-1}\right)$ & $-38.6 \pm 6.6$ & {$[-19.3]$} \\
\hline$\beta\left(\AA^{-1}\right)$ & 1.4 (fixed) & {$[2.4]$} \\
\hline & $2.3 \pm 0.4$ (free fit) & \\
\hline$r(\mathrm{~A})$ calculated & $\begin{array}{l}15 \pm 0.2(\beta \text { fixed at } 1.4) \\
9.5 \pm 2.1(\beta \text { unfixed })\end{array}$ & {$[8]$} \\
\hline $\begin{array}{l}r(\AA) \text { redox atoms }{ }^{c} \\
\mathrm{X} \text {-ray structure }\end{array}$ & $11-13$ & $11-13$ \\
\hline $\begin{array}{l}r(\AA) \text { edge- }- \text { edge }^{d} \\
\text { X-ray structure }\end{array}$ & 7 & 7 \\
\hline$\Delta H^{\#}\left(\mathrm{~kJ} \mathrm{~mol}^{-1}\right)$ & $24.5 \pm 1.2$ & $\begin{array}{c}23.1 \pm 8.2 \text { (1st slope) } \\
56.5 \pm 9.2(2 \text { nd slope })\end{array}$ \\
\hline$\Delta S^{\#}(\mathrm{~J} /(\mathrm{mol} \mathrm{K}))$ & $152 \pm 11$ & $\begin{array}{l}132.5 \pm 11.6(1 \text { st slope }) \\
19 \pm 2 \text { (2nd slope) }\end{array}$ \\
\hline$k_{\text {app }}$ at $25^{\circ} \mathrm{C}\left(\mathrm{s}^{-1}\right)$ & $2.9 \pm 0.1$ & $\begin{array}{l}81 \pm 1 \text { (phosphate absent) } \\
78 \pm 1 \text { (phosphate present) }\end{array}$ \\
\hline $\begin{array}{l}\text { net rate constant at } 25^{\circ} \mathrm{C}\left(\mathrm{s}^{-1}\right) \\
\text { ET reaction type } \\
\text { gating reactions }\end{array}$ & $\begin{array}{l}2.9 \pm 0.1 \\
\text { true }\end{array}$ & $\begin{array}{l}329 \pm 41 \\
\text { chemically gated } \\
\text { (i) formation of LThDP } \\
\text { (ii) decarboxylation of LThDP } \\
\text { (rate constants are given in Scheme 3) }\end{array}$ \\
\hline
\end{tabular}

${ }^{a}$ The reported errors represent the standard deviation from two independent experiments. ${ }^{b}$ The brackets indicate that these values are not physically meaningful because the Marcus theory may not be an appropriate formalism to describe the observed process. ${ }^{c}$ Distance between $\mathrm{C} 2$ of ThDP and the $\mathrm{N}_{1}$ and $\mathrm{N}_{5}$ atoms of the pyrazine/pyrimidine part of FAD. ${ }^{d}$ Distance between C2 of ThDP and the C7/C8 methyl groups of FAD.

effective through-bond pathway between the donor (HEThDP radical) and acceptor (flavin semiquinone), and it subsequently attacks the AcThDP intermediate formed upon this redox reaction. This mechanism, however, could apply only for the transfer of the second electron because transfer of the first electron is not affected by phosphate. Because such a discrimination is not expected for phosphate, we conclude that the wire mechanism is not a viable alternative.

The thermodynamic studies on the flavin cofactor demonstrated that the redox properties of FAD are also unaffected by phosphate. The determined midpoint potential of the flavin $\left(E_{\mathrm{m}}=-62 \mathrm{mV}\right.$ at $\left.\mathrm{pH} 6.0\right)$ is higher as that of free $\operatorname{FAD}\left(E_{\mathrm{m}}\right.$ $=-170 \mathrm{mV}$ at $\mathrm{pH} 6.0$ ). The thermodynamic stabilization of semireduced FAD radicals (55\%) is also independent of phosphate.

A mechanism that would be consistent with the experimental data is a kinetic coupling of phosphate and ET. A similar mechanism of CoA addition to the HEThDP radical was only recently suggested by Ragsdale and co-workers as one of several possible modes of action in PFOR catalysis (24). This would involve the transient formation of a highly reducing anion radical adduct. We thus envisage that phosphate attacks the kinetically stabilized HEThDP radical, forming a radical phosphate adduct as depicted in Scheme 3 . The negative charge of the resultant radical adduct would make it a low-potential intermediate, thus increasing the intrinsic driving force of the reaction. To achieve the observed $>10^{2}$-fold rate acceleration, the potential of the radical adduct would have to be $330 \mathrm{mV}$ lower than that of the HEThDP radical (see below). This is based on the assumption that a change of the intrinsic driving force solely accounts for the change of the ET rate, and all other Marcus parameters $\left(H_{\mathrm{AB}}\right.$ and $\left.\lambda\right)$ remain unaltered. In an alternative mechanism, phosphate might form an adduct with the
O-protonated HEThDP cation radical. The radical adduct would then undergo homolytic fragmentation to the $\mathrm{C} 2$ ylide and the O-protonated acetyl phosphate radical. Transfer of the second electron generates O-protonated acetyl phosphate, which then loses its proton to complete the reaction.

In the absence of phosphate, the slow radical decay appears to reflect a true ET reaction with an $H_{\mathrm{AB}}\left(16 \pm 5 \mathrm{~cm}^{-1}\right)$ that is below the limit for adiabatic reactions. The calculated reorganizational energy $(1.9 \pm 0.2 \mathrm{eV})$ is large but well within the range reported for the homologous reaction in PFOR (24) and in other ET reactions in proteins $(17,18$, $25)$. This large reorganizational energy and a relatively small intrinsic driving force $(-38.6 \pm 6.6 \mathrm{~kJ} / \mathrm{mol})$ manifest in the slow ET rates as the reaction is in the low driving force regime $\left(-\Delta G^{\circ} \ll \lambda\right)$. The calculated intrinsic driving force and the determined redox potential of the $\mathrm{FAD}_{\mathrm{sq}} / \mathrm{FAD}_{\text {red }}(-87$ $\pm 2 \mathrm{mV}$ ) couple allow an estimation of the redox potential of the HEThDP radical $(E=-487 \mathrm{mV})$. This value is slightly above that reported for a related nonenzymic hydroxyaryl thiazolium radical $(E=-560 \mathrm{mV}$, ref 26 ), inviting speculation that the enzyme active site may stabilize the radical intermediate and indicating a possible delocalization of the spin density of the unpaired electron in a $\pi$-type radical form. If we consider that the faster rate of the first ET from the enamine to the flavin (Scheme 3) was solely due to a larger intrinsic driving force of the first ET step when compared to the second transfer and that all other Marcus parameters $\left(H_{\mathrm{AB}}\right.$ and $\left.\lambda\right)$ would be comparable, the corresponding higher $\Delta G^{\circ}$ of $-70.4 \mathrm{~kJ} / \mathrm{mol}$ would translate into a $\Delta E$ of the enamine/radical and $\mathrm{FAD}_{\mathrm{ox}} / \mathrm{FAD}_{\mathrm{sq}}$ couples of approximately $720 \mathrm{mV}$. Using this theoretical approximation, the potential of the enamine can be estimated as -757 $\mathrm{mV}\left(E_{\mathrm{FAD} / \mathrm{FAD}_{\mathrm{sq}}}-\Delta E\right)$. The enamine at the active site would then be markedly stabilized because the $1 \mathrm{e}^{-}$oxidation 
Scheme 3: Proposed Model of the Kinetic Coupling Mechanism of Phosphate and ET in LpPOX Catalysis with Key Intermediates Invoked and Net Rate Constants Identified by Stopped-Flow Kinetics and a Rapid-Quench/ ${ }^{1} \mathrm{H}$ NMR Intermediate Analysis

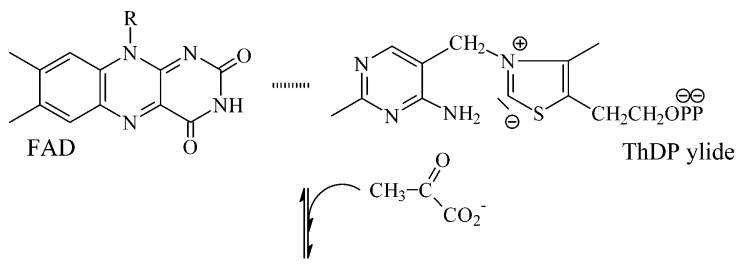

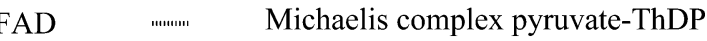
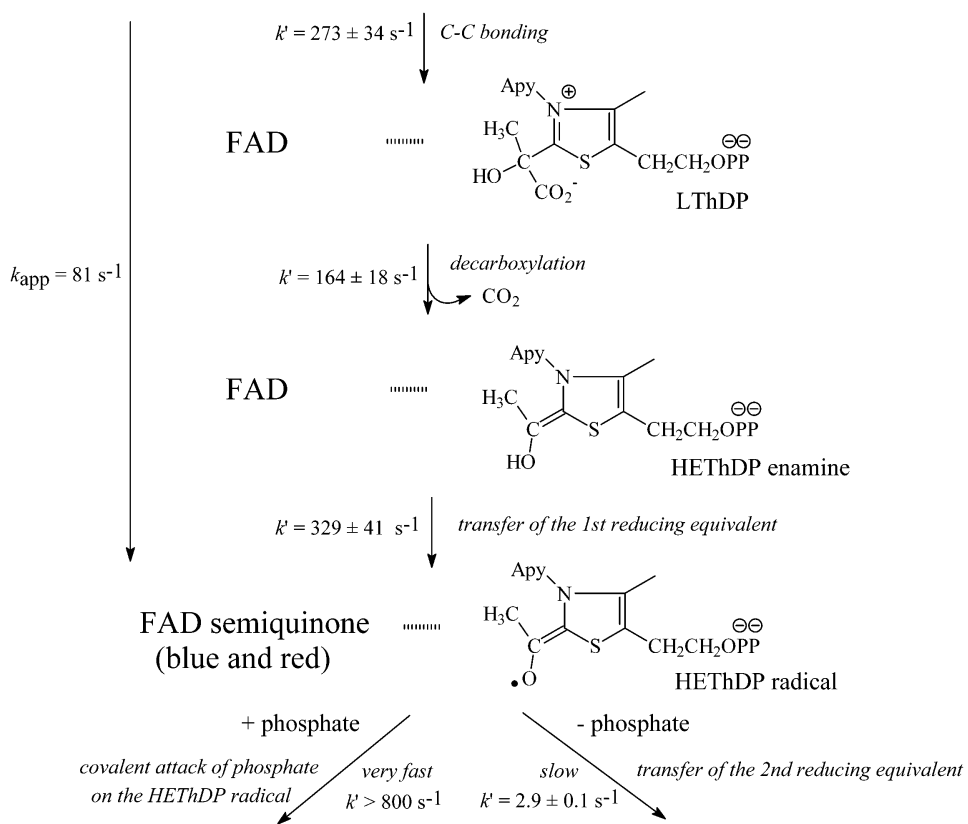

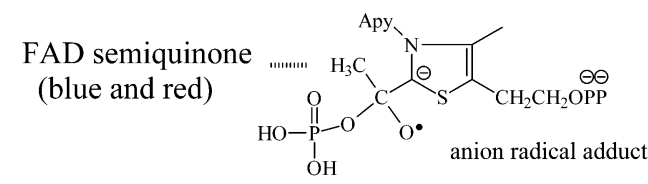

\begin{tabular}{l|l} 
transfer of the 2nd reducing equivalent & $\begin{array}{l}\text { very fast } \\
k^{\prime}>800 \mathrm{~s}^{-1}\end{array}$
\end{tabular}

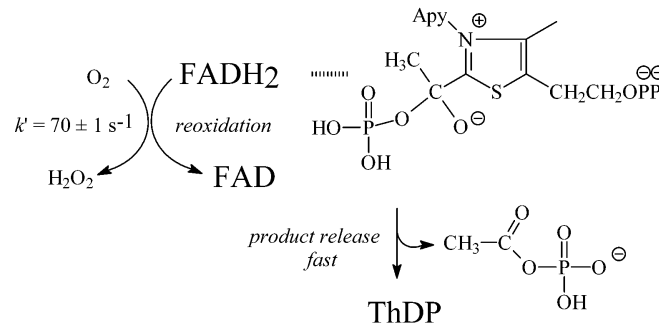

potential of a related, free thiazolium hydroxyaryl enamine is approximately $-970 \mathrm{mV}(26,27)$. Electrochemical studies on a number of different 2-alkyl-thiazolium enamines suggest higher potentials of these enamines; however, these studies do not include 2-hydroxyalkyl thiazolium compounds, the only ones that can be compared directly with the enzymatic intermediates (28). Even so, the systematic analysis of these compounds revealed that an oxygen substituent at the $\mathrm{C} 2 \alpha$ position (methoxy substituent) facilitates oxidation by 120 $\mathrm{mV}$ compared to a methyl substituent and by $250 \mathrm{mV}$ compared to a hydrogen substituent. A schematic comparison of these thermodynamic parameters is shown in Figure 9.

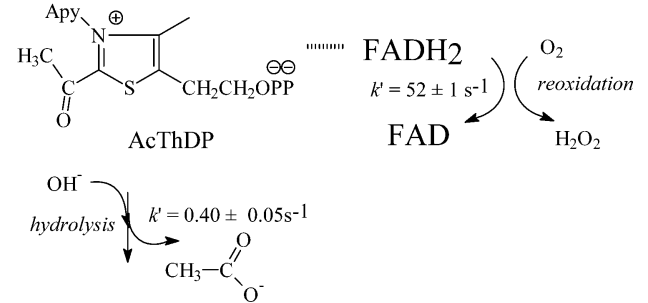

ThDP
Our data provide evidence that transfer of the two electrons from the central HEThDP enamine to the flavin occurs in a stepwise manner in POX. This supports conclusions from model studies on free thiaziolum-derived enamines and flavins (29). The fine-tuned intrinsic thermodynamic properties of the flavin and thiamin enamine/radical intermediates in $L p \mathrm{POX}$ and a large reorganizational energy enforce the lifetime of the transient hydroxyethyl-ThDP radical to be sufficient for a nucleophilic attack of phosphate onto this highly reactive intermediate. In a study yet to be published, we did obtain evidence that also other nucleophiles easily add to the HEThDP radical, thereby promoting further 

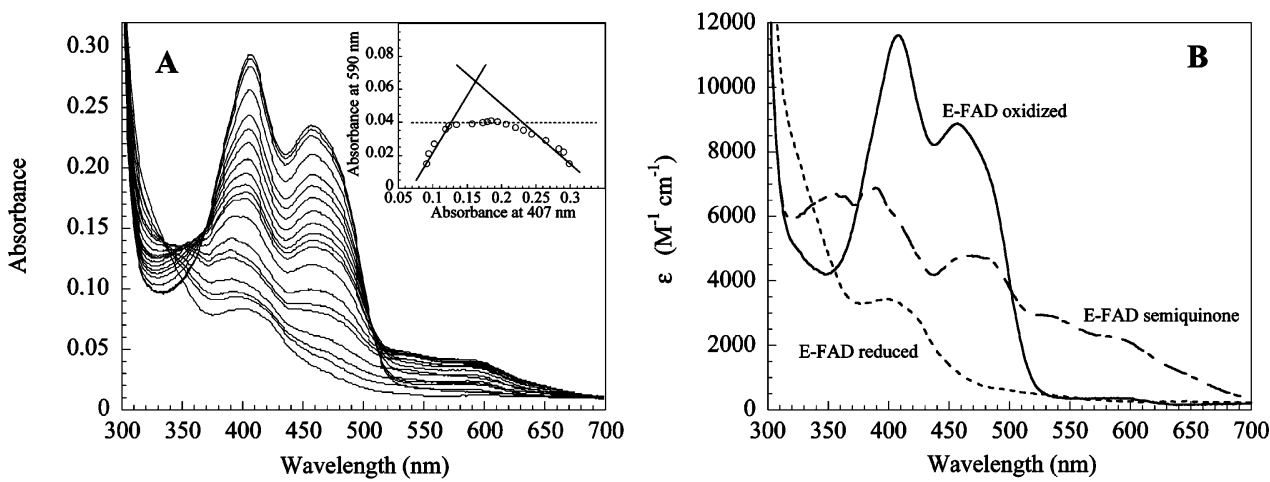

FIGURE 7: Absorption spectra of $L p P O X$ during reductive titration using sodium dithionite as a reductant under anaerobic conditions and deconvoluted spectra of the oxidized, semireduced, and fully reduced enzyme. (A) Solution of $24 \mu \mathrm{M} L p$ POX (active-site concentration) in $0.2 \mathrm{M}$ potassium phosphate buffer at $\mathrm{pH} 6.0$ was reduced by the stepwise addition of sodium dithionite under anaerobic conditions at 15 ${ }^{\circ} \mathrm{C}$. (Inset) Maximum semiquinone formation (thermodynamic stabilization) was estimated by plotting the changes in absorbance at the maximum wavelength of the semiquinone $(590 \mathrm{~nm})$ versus the oxidized form $(407 \mathrm{~nm})$. (B) Spectra of the oxidized, semireduced, and fully reduced FAD in $L p$ POX obtained by Eigenfactor analysis using Specfit32.
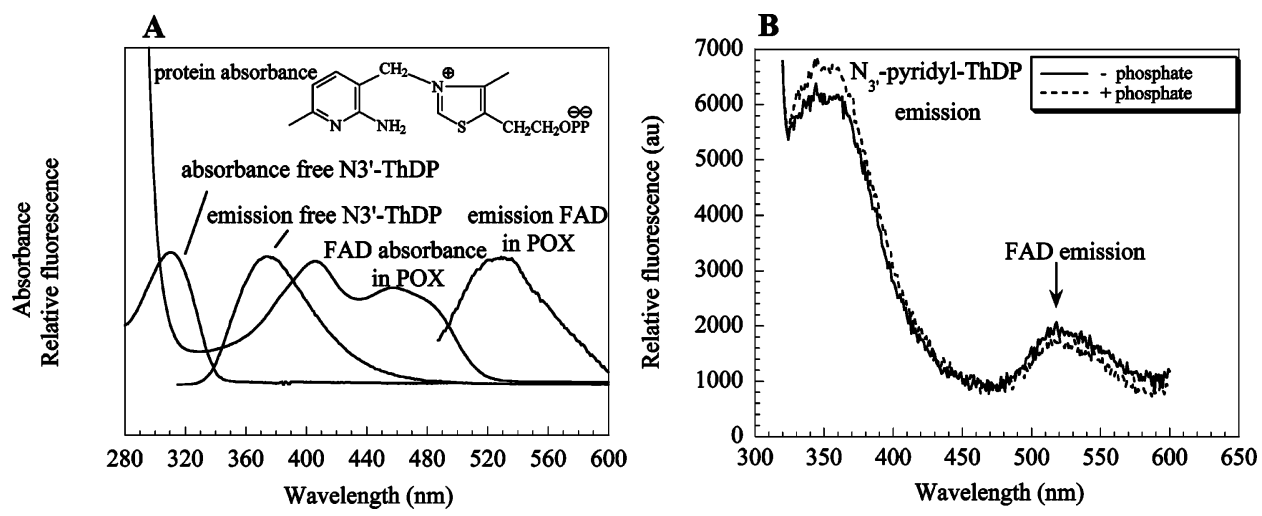

FIGURE 8: Absorbance and emission spectra of $L p P O X$ species relevant for FRET studies. (A) Schematic representation of the UV-vis absorbance and fluorescence emission spectra of the putative donor/acceptor pair N3'-pyridyl-ThDP and FAD. (Inset) Chemical structure of N3'-pyridyl-ThDP. (B) Fluorescence emission spectra of enzyme-bound FAD in LpPOX (in either 0.2 M potassium phosphate buffer at pH 6.0 or $0.1 \mathrm{M}$ Mes/NaOH at pH 6.0) upon excitation at $315 \mathrm{~nm}$ (absorbance of enzyme-bound N3'-pyridyl-ThDP).

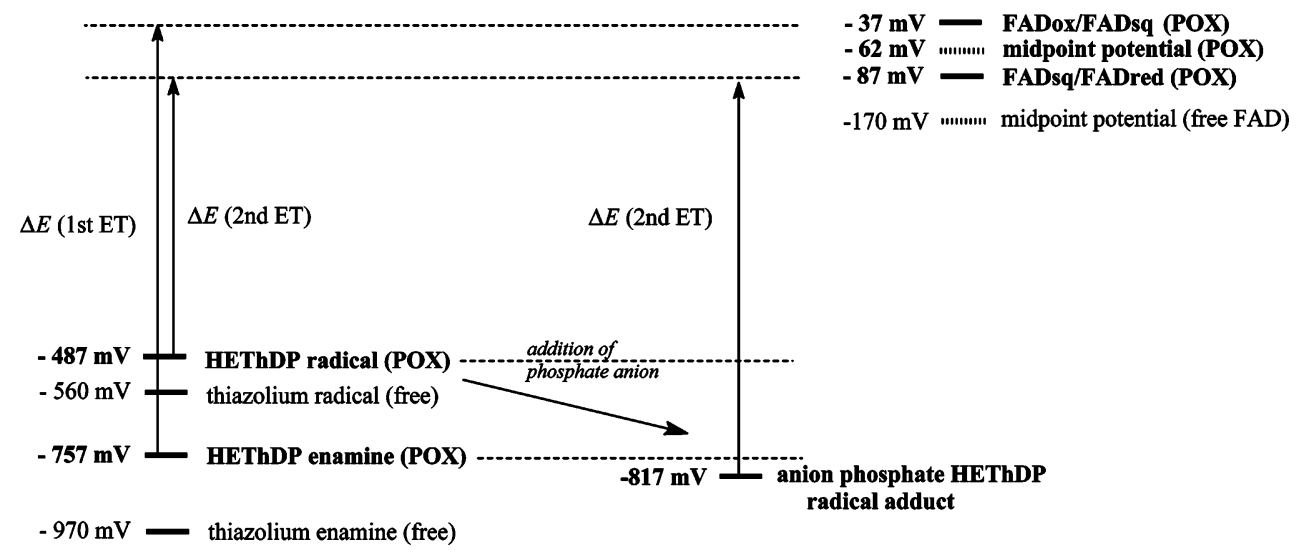

FIGURE 9: Diagrammatic representation of the redox potentials estimated for ThDP, intermediates, and enzyme-bound FAD in $L p P O X$. The data are as obtained from Figures 6 and 7 and Figure S2 in the Supporting Information. Note that the potential of the HEThDP enamine and the phosphate-HEThDP radical adducts have been calculated under the assumption that only a change of the intrinsic driving force accounts for faster ET from the HEThDP enamine and the proposed phosphate-HEThDP radical adduct when compared to the slow HEThDP radical decay in the absence of phosphate. Thus, these values may not be valid when $\lambda$ and $H_{\mathrm{AB}}$ are also affected (see the text).

reduction of the FAD semiquinone (manuscript in preparation).

Aside PFOR and $L p$ POX, we have neither spectroscopic indications for transient radical intermediates nor evidence for a role of phosphate in ET reactions in other ThDPdependent flavoenzymes such as AHAS or $E c$ POX. In these two enzymes, however, there is no need for a kinetically stabilized HEThDP radical because no "specific" nucleo- philes such as CoA (PFOR) or phosphate ( $L p \mathrm{POX}$ ) are to be added to the intermediates. Therefore, we conclude that the FeS clusters in PFOR and the flavin in $L p$ POX are not only suited as charge conductors of low-potential electrons but also elegantly concur in the stabilization of highly reactive thiamin radical intermediates. In this context, $L p$ POX constitutes the first example of a radical-based coupling mechanism of phosphate and ET. 


\section{SUPPORTING INFORMATION AVAILABLE}

The ${ }^{1} \mathrm{H}$ NMR spectroscopic intermediate studies on POX shown in Figure $\mathrm{S} 1$ and the experimental data for the estimation of the midpoint potential of the enzyme-bound FAD given in Figure S2. This material is available free of charge via the Internet at http://pubs.acs.org.

\section{REFERENCES}

1. Muller, Y. A., and Schulz, G. E. (1993) Structure of the thiamineand flavin-dependent enzyme pyruvate oxidase, Science 259, 965967.

2. Sedewitz, B., Schleifer, K. H., and Götz, F. (1984) Purification and biochemical characterization of pyruvate oxidase from $\mathrm{Lac}$ tobacillus plantarum, J. Bacteriol. 160, 273-278.

3. Gruys, K. J., Halkides, C. J., and Frey, P. A. (1987) Synthesis and properties of 2-acetylthiamin pyrophosphate: An enzymatic reaction intermediate, Biochemistry 26, 7575-7585.

4. Lienhard, G. E. (1966) Kinetics and mechanism of the hydrolysis of 2-acetyl-3,4-dimethylthiazolium ion, J. Am. Chem. Soc. 88 , 5642-5649.

5. Ragsdale, S. W. (2003) Pyruvate ferredoxin oxidoreductase and its radical intermediate, Chem. Rev. 103, 2333-2346.

6. Menon, S., and Ragsdale, S. W. (1997) Mechanism of the Clostridium thermoaceticum pyruvate:ferredoxin oxidoreductase: Evidence for the common catalytic intermediacy of the hydroxyethylthiamine pyropyrosphate radical, Biochemistry 36 , 8484-8494.

7. Golbik, R., Neef, H., Hübner, G., König, S., Seliger, B., Meshalkina, L., Kochetov, G. A., and Schellenberger, A. (1991) Function of the aminopyrimidine part in thiamine pyrophosphate enzymes, Bioorg. Chem. 19, 10-17.

8. Wille, G., Ritter, M., Friedemann, R., Mäntele, W., and Hübner, G. (2003) Redox-triggered FTIR difference spectra of FAD in aqueous solution and bound to flavoproteins, Biochemistry 42, $14814-14821$

9. Tittmann, K., Proske, D., Spinka, M., Ghisla, S., Rudolph, R., Hübner, G., and Kern, G. (1998) Activation of thiamin diphosphate and FAD in the phosphate dependent pyruvate oxidase from Lactobacillus plantarum, J. Biol. Chem. 273, 12929-12934.

10. Tittmann, K., Schröder, K., Golbik, R., McCourt, J., Kaplun, A., Duggleby, R. G., Barak, Z., Chipman, D. M., and Hübner, G. (2004) Electron transfer in acetohydroxy acid synthase as a side reaction of catalysis. Implications for the reactivity and partitioning of the carbanion/enamine form of ( $\alpha$-hydroxyethyl)-thiamin diphosphate in a "nonredox" flavoenzyme, Biochemistry 43, 8652-8661.

11. Bradford, M. M. (1976) A rapid and sensitive method for the quantitation of microgram quantities of protein utilizing the principle of protein-dye binding, Anal. Biochem. 72, 248-254.

12. Tittmann, K., Golbik, R., Ghisla, S., and Hübner, G. (2000) Mechanism of elementary catalytic steps of pyruvate oxidase from Lactobacillus plantarum, Biochemistry 39, 10747-10754.

13. Gibson, Q. H., Swoboda, B. E., and Massey, V. (1964) Kinetics and mechanism of action of glucose oxidase, J. Biol. Chem. 239, $3927-3934$
14. Marcus, R. A., and Sutin, N. (1985) Electron transfers in chemistry and biology, Biochim. Biophys. Acta 811, 265-322.

15. Winkler, J. R., and Gray, H. B. (1992) Electron transfer in ruthenium-modified proteins, Chem. Rev. 92, 369-379.

16. Moser, C. C., Keske, J. M., Warncke, K., Farid, R. S., and Dutton, P. L. (1992) Nature of biological electron transfer, Nature 355, 796-802.

17. Davidson, V. L. (2002) Chemically gated electron transfer. A means of accelerating and regulating rates of biological electron transfer, Biochemistry 41, 14633-14636.

18. Page, C. C., Moser, C. C., Chen, X., and Dutton, P. L. (1999) Natural engineering principles of electron tunnelling in biological oxidation-reduction, Nature 402, 47-52.

19. Tittmann, K., Golbik, R., Uhlemann, K., Khailova, L., Schneider, G., Patel, M., Jordan, F., Chipman, D. M., Duggleby, R. G., and Hübner, G. (2003) NMR analysis of covalent intermediates in thiamin diphosphate enzymes, Biochemistry 42, 7885-7891.

20. Clark, W. M. (1960) Oxidation-Reduction Potentials of Organic Systems, Williams and Wilkins, Baltimore, MD.

21. Gampp, H., Maeder, M., Meyer, C. J., and Zuberbühler, A. D. (1986) Calculation of equilibrium constants from multiwavelength spectroscopic data-IV. Model-free least-squares refinement by use of evolving factor analysis, Talanta 33, 943-951.

22. Zhang, S., Liu, M., Zhang, Z., and Jordan, F. (2004) C2- $\alpha-$ lactylthiamin diphosphate is an intermediate on the pathway of thiamin diphosphate-dependent pyruvate decarboxylation. Evidence on enzymes and models, J. Biol. Chem. 279, 54312-54318.

23. Bertagnolli, B. L., and Hager, L. P. (1991) Activation of Escherichia coli pyruvate oxidase enhances the oxidation of hydroxyethylthiamin pyrophosphate, J. Biol. Chem. 266, 1016810173.

24. Furdui, C., and Ragsdale, S. W. (2002) The roles of coenzyme A in the pyruvate:ferredoxin oxidoreductase reaction mechanism rate enhancement of electron transfer from a radical intermediate to an iron-sulfur cluster, Biochemistry 41, 9921-9937.

25. Wilson, E., Mathews, F. S., Packman, L. C., and Scrutton, N. S (1995) Electron tunneling in substrate-reduced trimethylamine dehydrogenase: Kinetics of electron transfer and analysis of the tunneling pathway, Biochemistry 34, 2584-2591.

26. Nakanishi, I., Itoh, S., Suenobu, T., Inoue, H., and Fukuzumi, S (1997) Redox behavior of active aldehydes derived from thiamin coenzyme analogs, Chem. Lett., 707-708.

27. Nakanishi, I., Itoh, S., Suenobu, T., Inoue, H., and Fukuzumi, S (1998) Direct observation of radical intermediates while investigating the redox behavior of thiamin coenzyme models, Angew. Chem. Int. Ed. 37, 992-994.

28. Barletta, G., Chung, A. C., Rios, C. B., Jordan, F., and Schlegel, J. M. (1990) Electrochemical oxidation of enamines related to the key intermediate on thiamin diphosphate dependent enzymic pathways: Evidence for one-electron oxidation via a thiazolium cation radical, J. Am. Chem. Soc. 112, 8144-8149.

29. Chiu, C. C., Pan, K., and Jordan, F. (1995) Modeling an elementary step of the enzyme pyruvate oxidase: Oxidation of a thiamin diphosphate-bound enamine intermediate by a flavin analog, $J$. Am. Chem. Soc. 117, 7027-7028. 